\title{
$\underline{\text { Nuevas estrategias de revitalización del quichua santiagueño }}$
}

\author{
Lelia Inés Albarracín
}

\section{SciELO Books / SciELO Livros / SciELO Libros}

ALBARRACÍN, L. I. Nuevas estrategias de revitalización del quichua santiagueño. In: HABOUD BUMACHAR, M., SÁNCHEZ AVENDAÑO, C., and GARCÉS VELÁSQUEZ, F., eds. Desplazamiento lingüístico y revitalización: reflexiones y metodologías emergentes [online]. Quito: Editorial Abya-Yala, 2020, pp. 63-78. Desafíos en la Diversidad collection, n. 2. ISBN: 978-9978-10-541-2. http://doi.org/10.7476/9789978105726.0004.

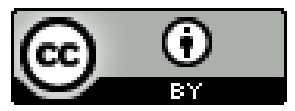

All the contents of this work, except where otherwise noted, is licensed under a Creative Commons Attribution 4.0 International license.

Todo o conteúdo deste trabalho, exceto quando houver ressalva, é publicado sob a licença Creative Commons Atribição 4.0.

Todo el contenido de esta obra, excepto donde se indique lo contrario, está bajo licencia de la licencia Creative Commons Reconocimento 4.0. 


\title{
Nuevas estrategias de revitalización del quichua santiagueño
}

Lelia Inés Albarracín ${ }^{1}$

\begin{abstract}
Resumen
Los procesos de revitalización lingüístico-cultural son muy diversos y de región en región van cambiando las problemáticas consideradas y las estrategias para su abordaje. Hay situaciones en las cuales las comunidades de hablantes han logrado tomar el control de los mismos y los conducen con menor o mayor suerte, pero hay otras situaciones en las que dichos procesos se encuentran con barreras casi infranqueables y que exigen un considerable esfuerzo de pertinacia y perseverancia para lograr superarlas. Uno de los escenarios posibles, es un contexto adverso, en donde el Estado está ausente y la sociedad es indiferente frente al destino de las lenguas amenazadas, circunstancia que se ve agravada cuando se trata de grupos sociales subalternizados, de comunidades campesinas o de pueblos originarios con procesos de reetnización aún muy incipientes, que suelen estar dispersos o aislados geográficamente. Incluso hay realidades aún más complejas, tal es el caso de la comunidad quichuahablante de Santiago del Estero, cuya población mayoritariamente es criolla, pero habla una lengua indígena y con procesos de reemergencia étnica en diversas zonas del territorio provincial, algunos de ellos reivindicando lenguas desaparecidas de las que nada se sabe o de improbada existencia.

En este trabajo describo acciones de revitalización que se llevan a cabo en la provincia de Santiago del Estero desde la institución universitaria, con una reflexión crítica acerca de ellas, de su implementación y de las nuevas estrategias seguidas en la búsqueda de soluciones propias - más dinámicas - que permitan a los hablantes de las lenguas minorizadas, diseñar y ejecutar acciones destinadas a perforar las barreras de la discriminación lingüística.
\end{abstract}

\section{Introducción}

Los procesos de revitalización lingüístico-cultural de las lenguas amerindias en términos generales persiguen el objetivo de preservar las mismas del riesgo de extinción. También de manera general podríamos decir que las lenguas comienzan su proceso de extinción cuando se produce la interrupción de la transmisión intergeneracional (Alderetes \& Albarracín, 2004, p. 86). Planteadas, así

1 Universidad Nacional de Santiago del Estero, Argentina. 
las cosas, la muerte de lenguas parece conducir al sencillo esquema: enfermedad=interrupción de la transmisión, remedio=revitalización. Sin embargo, sabemos que los factores que inciden en la desaparición de una lengua son de naturaleza muy diversa y responden, principalmente, a causas socio-políticas, socio-económicas y socioculturales. De allí que el diagnóstico de las razones por las que los padres no transmiten la lengua a sus hijos nos termina enfrentando a una multiplicidad de factores, algunos de ellos de muy antigua data, entroncados con la historia misma de cada región/comunidad. De hecho, fenómenos sociolingüísticos actuales, considerados "modernos", encuentran su explicación y justificación en lo más profundo de la historia socio-cultural de cada comunidad. Frente a esta notable diversidad de factores causales de la interrupción intergeneracional, es esperable que los procesos de revitalización lingüístico-cultural también sean muy diversos, según las diferentes estrategias empleadas para el abordaje de las problemáticas específicas consideradas para cada región/comunidad.

Hacia fines del siglo pasado y en la primera década del presente, nuestras investigaciones científicas permitieron realizar una descripción lingüística formal autónoma de la lengua quichua ${ }^{2}$ con respecto al castellano y diseñar una metodología de enseñanza bilingüe desligada de representaciones sociolingüísticas provenientes de una visión hispánica de la lengua. El paso siguiente fue dar inicio, desde la institución universitaria, a acciones concretas de revitalización a favor de la lengua indígena, que se encuentran en pleno desarrollo y cuya evolución está siendo monitoreada de manera regular.

2 La quichua (en femenino) es el nombre del dialecto quechua del grupo QII-C, en la clasificación de Torero, que se habla en la provincia de Santiago del Estero. Los quichuahablantes se autodenominan a sí mismos como "quichuistas". Durante la primera década del siglo XXI se publicaron tres gramáticas descriptivas (Alderetes, 2001; Nardi, 2002; Albarracín, 2009, 2011). 


\section{Los procesos de revitalización}

Hasta comienzos del siglo XXI no hubo en Santiago del Estero procesos de revitalización lingüística concebidos como tales, en el marco de una planificación lingüística. El único antecedente digno de mención ocurrió en la década de los años 80 del siglo pasado, cuando hubo un frustrado intento de implementar la enseñanza de la lengua quichua como asignatura especial en tres escuelas rurales, pero la iniciativa no logró consolidarse ni prosperar (Sueldo, 2014, p. 554).

Analizando experiencias vividas en otros países vimos que había situaciones en las cuales las comunidades de hablantes habían logrado tomar el control de los procesos de revitalización y los condujeron con menor o mayor suerte, pero también había otras situaciones en las que dichos procesos se encontraron con barreras casi infranqueables y que exigieron un considerable esfuerzo de pertinacia y perseverancia para intentar superarlas. La situación de Santiago del Estero se inscribía en este segundo escenario posible, en un contexto adverso, en donde el Estado estaba ausente y la sociedad era indiferente frente al destino de las lenguas amenazadas, circunstancia que se veía agravada cuando se trataba de grupos sociales subalternizados, de comunidades campesinas o de pueblos originarios, que estaban dispersos o aislados geográficamente.

Incluso la realidad es aún más compleja, puesto que la comunidad quichuahablante de Santiago del Estero, es una población campesina mayoritariamente criolla, que habla una lengua indígena pero que no se autorreconoce como indígena y que observa con cierto grado de desconfianza, los incipientes procesos de reemergencia étnica en diversas zonas del territorio provincial, algunos de ellos reivindicando lenguas extinguidas de las que nada se sabe. Al respecto, Alderetes (2011, p. 85) señala: 
Nos interesa plantear el caso de aquellos grupos étnicos que se consideraban cultural y lingüísticamente extinguidos, y que 'emergen' procurando recuperar un pasado asumido como propio, pero cuyas acciones de autoadscripción étnica aparecen como escindidas de la tradición cultural reclamada e incluso algunas referencias históricas invocadas no guardan continuidad con la memoria histórica de las propias comunidades.

El hecho de que aspectos históricos y culturales ajenos a una región, en algunos casos tomados de fuentes no indígenas, sean introducidos de manera forzada, tanto por particulares como por organizaciones e incluso por organismos del Estado, y la utilización de textos carentes de todo rigor científico, ha generado un intenso debate acerca de la legitimidad de estos casos de autodefinición étnica. Pero lo que nos interesa de estos procesos es que su emergencia contemporánea constituye un nuevo referente para las políticas públicas en contextos multiculturales, especialmente las relacionadas con la educación.

Con este panorama y ya en el presente siglo, el primer paso fue la implementación de una carrera universitaria en relación directa con la lengua y su enseñanza, para desarrollar profesionales con una sólida formación, comprometidos con el desarrollo de su pueblo y la región, con capacidad de análisis propio, que le permita tomar las decisiones necesarias y practicar la iniciativa creadora para impulsar actividades que contribuyan a promover procesos de revitalización lingüístico-cultural. Esta carrera, la Tecnicatura Superior en Educación Intercultural Bilingüe con Mención en Lengua Quichua, que dictamos en la Universidad Nacional de Santiago del Estero, ha posibilitado con la participación de sus egresados, alumnos y docentes, diseñar y ejecutar acciones concretas tendientes a promover y revitalizar la lengua quichua (Albarracín, 2018).

La dependencia de los procesos de revitalización respecto de factores sociales, económicos y políticos e incluso de hechos históri- 


\section{7}

cos, determina que estrategias que fueron exitosas en una región, al ser replicadas en otra no alcancen los mismos resultados. Cuando se habla de "nuevas" estrategias de revitalización, por lo general se piensa en las TIC, en la utilización de productos tecnológicos de última generación, la implementación de innovadores proyectos pedagógicos y, sin embargo, a veces lo "nuevo" nos conduce a lo que ya conocemos, pero planteado desde una perspectiva liberadora, descolonizadora, que promueva acciones concretas para movilizar a una comunidad en favor de su lengua. Cito por caso, un programa de radio de una FM local de Villa Salavina, en donde su locutor, egresado de nuestros cursos, no se limita a usar la lengua quichua en sus intervenciones, sino que ha convertido su cabina de transmisión en un punto de encuentro, en donde jóvenes y adultos de la comunidad se dan cita para salir al aire con su voz, interpelando a sus vecinos para que no cejen en el uso cotidiano de su lengua y en la enseñanza a los niños.

\section{La documentación lingüística}

Un factor relevante en los procesos de mantenimiento y revitalización de las lenguas indígenas es el mantener de manera constante, los proyectos de documentación de las mismas. Me refiero a una tarea sistemática y planificada de actualización permanente de los registros y datos obtenidos, simultánea con el resguardo de la información histórica. No falta quienes creen que esta tarea se agota en la publicación de una gramática y un diccionario únicos y que las descripciones gramaticales y el léxico recogido permanecen inalterables con el tiempo, sin advertir que solo representan una "fotografía instantánea" en la historia lingüística de una determinada lengua. Por otro lado, el interés por los archivos de la lengua no es realizar "arqueología lingüística" para establecer un "museo filológico", sino por el rol que juegan esos archivos en la actualidad, frente a la amenaza de extinción de la len- 
gua, como insumo para la investigación lingüística contemporánea y para la definición de políticas lingüísticas (Albarracín, 2013).

En el ámbito de la cátedra a mi cargo en la universidad, se vienen realizando registros digitalizados que constituyen un corpus sumamente valioso y que ya han permitido publicaciones tales como gramáticas descriptivas, diccionarios y antologías. Estas actividades no solo contribuyen a un mejor conocimiento de esta variedad, sino que también representan un aporte concreto en el plano educativo. Si bien las dos primeras gramáticas publicadas estaban acompañadas, respectivamente, de un diccionario quichua-castellano y castellano-quichua, muy pronto las actividades de docencia, investigación y extensión hicieron sentir la necesidad de contar con un nuevo diccionario, integrado en un único volumen. Esta última publicación busca, por un lado, actualizar la investigación de la lengua; pero además es visto como una herramienta para resistir a la presión de la cultura castellanizante impulsada desde la sociedad hegemónica. Cuanto más valiosa es esa herramienta, en las manos de un campesino a quien durante siglos se le ha transmitido la idea de que "la quichua no se escribe, se habla".

Si bien reconocemos que un estudio filológico puede parecer una preocupación casi exquisita frente a los graves problemas sociales de exclusión y marginalidad que viven los quichuahablantes, también es cierto que el estudio diacrónico de una lengua amenazada de extinción es una tarea necesaria e impostergable. Tarea que hemos asumido con la convicción de que tiene que ver con testimoniar nuestra identidad y saber y poder responder cuando nos pregunten qué hicimos con nuestra riqueza cultural y nuestro patrimonio lingüístico.

Sobre la base de estas consideraciones, el diseño del nuevo producto perseguía entre sus propósitos, crear actitudes lingüísticas positivas en la formación de técnicos y docentes y promover un posicionamiento ideológico de lealtad hacia la lengua indígena. 


\section{9}

Desde la perspectiva del usuario de los diccionarios previos, habíamos advertido dos inconvenientes: los hispanoparlantes no lograban advertir las diferencias semánticas como, por ejemplo, las existentes entre los verbos sutiyachiy y waqyay ambos traducibles por "llamar". A su vez, los quichuahablantes tendían a utilizar voces quichuas, pero asignándoles el significado castellano, como, por ejemplo, usar con el sentido de "idioma" a la voz qallu que designa a la lengua como órgano. Nos quedaba claro que la sola ejemplificación no resultaba suficiente y de esta apreciación fue madurando la idea de construir un diccionario temático, algo inexistente para esta lengua.

La primera tarea fue definir las áreas temáticas, notoriamente diferentes a las de los diccionarios de lenguas indoeuropeas, no solo debido a las diferentes cosmovisiones, sino también al contexto socio-cultural en que la lengua se desarrolla. Luego se procedió a digitalizar la información, codificando cada entrada lexical conforme a las áreas y subáreas preestablecidas. Por último, mediante la utilización de filtros, se fueron agrupando las entradas para verificar la pertinencia de las áreas asignadas. Al mismo tiempo, se fueron redefiniendo algunas subáreas para lograr una mejor descripción.

Si bien la idea original era un volumen con dos secciones, una con un listado alfabético y otra con listados por áreas, razones presupuestarias obligaron a imprimir en formato de libro sólo la sección alfabética. De todos modos, en dicha sección cada entrada también lleva indicada el área y las subáreas correspondientes.

Con esta publicación, realizada por la Editorial Humanitas de la Facultad de Filosofía y Letras de la Universidad Nacional de Tucumán (ver Figura 1) y prologado por la Dra. Marleen Haboud, por primera vez los usuarios cuentan con un diccionario bilingüe de términos usuales y cuyas contrapartes quichua-castellano y castellano-quichua guardan entre sí una relación biunívoca. 


\section{Figura 1}

Nuevo Diccionario Temático Quichua-Castellano Castellano-Quichua, Editorial Humanitas (2017)

Lelia Inés Albarracín

NUEVO DICCIONARIO TEMÁTICO

QUICHUA-CASTELLANO / CASTELLANO QUICHUA

El presente diccionario recoge el léxico de la lengua quichua que se habló en una extensa región del Noroeste Argentino y que aún se conserva en la provincia de Santiago del Estero. El mismo apunta a satisfacer las necesidades de lingüistas y profesionales de otras disciplinas, como así también de estudiantes y egresados de las carreras relacionadas con esta lengua, de docentes de las zonas bilingües de dicha provincia y del público en general. Al respecto, Marleen Haboud señala: "No hay duda que Lelia Inés Albarracín ha escrito cada línea con un profundo conocimiento, cariño y compromiso con los hablantes, y con la convicción de que su aporte motivará a hablantes y no hablantes a buscar mejores días para las lenguas minorizadas que sobreviven siempre en zozobra. Que nos aliente, esta obra, a vivir la diversidad y la diferencia plenamente y contodos nuestros sentidos"

Calección DIÁLOGOS

A través de la colección "Diálogos" la Facultad de Filosofia y Letras public

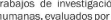

Lelia Inés Albarracín NUEVO DICCONARIOTEMÁTICO QUICHUA-CASTELLANO CASTELLANO-QUICHUA

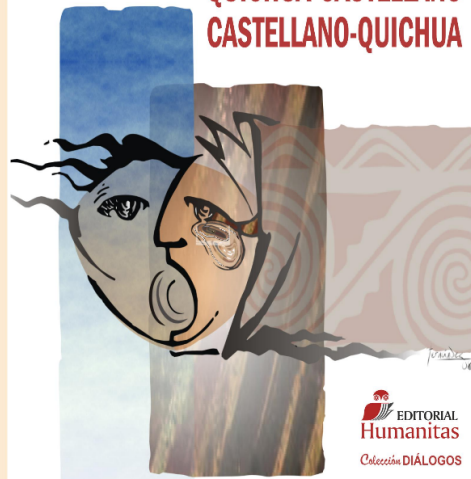

De acuerdo con los testimonios que hemos recogido por parte de lectores jóvenes quichuahablantes, este diccionario les está permitiendo reconocer expresiones que escucharon de sus mayores, cuyo significado desconocían y que no se atrevieron a preguntar; a la vez, les permite despejar sus dudas sobre ciertos términos y afianzar el uso de los mismos. El entusiasmo que ha despertado esta nueva publicación entre algunos quichuahablantes, los ha motivado a hacernos llegar, espontáneamente, contribuciones en cuanto a nuevos lexemas, variantes fonológicas, extensiones y precisiones de campos semánticos, etc., que permiten enriquecer la base de datos léxica que disponemos.

En el caso de los lectores hispanoparlantes, el beneficio es obvio. Téngase presente que el único diccionario castellano-quichua 
que se disponía hasta hace relativamente poco tiempo, era el publicado en 1977 por Domingo Bravo.

Quienes se han apropiado de manera inmediata de esta herramienta, han sido los alumnos y egresados de nuestra carrera universitaria sobre educación intercultural bilingüe. Sus intervenciones en las redes sociales y en las comunicaciones por medio de los servicios de mensajería de dispositivos móviles, han servido de estímulo a una creciente participación de hablantes que no estaban habituados a escribir en su propia lengua y que hoy lo realizan sobre una nutrida temática con una variada tipología textual.

Aquí la novedad estratégica no es el uso de dispositivos electrónicos sino la tarea pedagógica que nuestros alumnos y egresados realizan, de manera espontánea y sin imposiciones, a través de la recreación y conservación del léxico y en la adquisición del alfabeto normalizado por parte de personas cuya lengua ha sido ignorada por el sistema educativo. De este modo, por las redes están circulando, en lengua indígena, diálogos, canciones, chistes, relatos breves, reclamos sociales, comentarios políticos, consignas, etc., manifestaciones que permiten superar la vergüenza y la discriminación y que constituyen una evidente contribución a la revitalización de una lengua. Es notorio también el incremento de las publicaciones con una intención identitaria.

El entusiasmo por escribir ha abierto un nuevo canal de comunicación entre padres e hijos, cito como ejemplo ilustrativo el caso de jóvenes de Villa Salavina, alumnos de $5^{\circ}$ año del Colegio Secundario "Konrad Adenauer", quienes han ejecutado un proyecto educativo que revaloriza la escritura en lengua quichua, con el apoyo de alumnos de la Tecnicatura de la Universidad Nacional de Santiago del Estero a través del voluntariado universitario ${ }^{3}$. El resultado es el

3 El Voluntariado Universitario es un programa, creado en el año 2006, dependiente del Ministerio de Educación de la Nación que financia proyectos de extensión con 
libro bilingüe "Salavinamanta Mosqos", de confección artesanal, que contiene textos producidos por los jóvenes consistentes en anécdotas, historias de vida, relatos sobre diferentes vivencias y poesías que describen el diario vivir de toda la comunidad salavinera, como así también el pensamiento de los jóvenes que buscan alternativas para su futuro. Para garantizar la calidad de las transcripciones los jóvenes han contado con el apoyo de los mayores, tanto docentes como padres y abuelos.

La dinámica de los procesos sociales en las comunidades subalternizadas incide, a favor o en contra, sobre las acciones de revitalización lingüística. Está en la inteligencia de quienes trabajan en proyectos relacionados con la temática, el advertir tempranamente la necesidad de corregir el rumbo cuando no se están obteniendo los resultados esperados. Replantear las estrategias no implica, necesariamente, recurrir a más y mejor tecnología, a sofisticados soportes lógicos o a nuevos paradigmas pedagógicos. A veces, sólo basta con saber escuchar, tener espíritu autocrítico y reconocer nuestras propias limitaciones, para recuperar "oxidadas" herramientas que siguen teniendo plena vigencia y que, injustamente, fueron consideradas obsoletas. Recuerdo una anécdota vivida precisamente en Villa Salavina, en ocasión de una visita. Un vecino, a quien acabábamos

el objetivo de posibilitar la participación responsable y solidaria de la comunidad universitaria en articulación con los distintos actores de la sociedad civil, como así también la incorporación de espacios donde pueda ponerse a prueba en situación auténtica, las habilidades y conocimientos teóricos adquiridos, como prácticas centrales en la formación de un profesional íntegro. En los proyectos que fueron aprobados y ejecutados desde nuestra carrera, se ha procurado siempre generar una concienciación transformadora, tanto en los voluntarios como en los miembros de la comunidad quichuahablante hacia la cual se dirigen las acciones de voluntariado. Es una herramienta útil, pero que debe ser usada con criterio para no sobrecargar al estudiante con actividades que insuman un tiempo considerable que vaya en detrimento de su rendimiento académico. 
de conocer, nos invitó a pasar al interior de su casa porque deseaba mostrarnos su tesoro más preciado, al cabo de unos instantes regresó ceremonioso como si portara una joya entre sus manos: un libro. Y es que, en estas comunidades, en donde el analfabetismo registra los porcentajes más altos de toda la República Argentina, el libro sigue siendo el símbolo de un derecho negado, de un territorio a recuperar. He aquí la importancia simbólica de la escritura de la cual habla Nila Vigil (2004, p. 176). Hemos sido testigos privilegiados de la intensa emoción de los quichuistas al ver reflejados sus rostros en las tapas, encontrar sus nombres en el interior y sentir respirar su lengua y su cultura en cada página de los libros que hemos logrado editar.

Pero no todas son buenas noticias, también se observa una creciente actividad de personas e incluso pequeñas agrupaciones, que constantemente descalifican a quienes utilizan el alfabeto normalizado e instan a seguir usando el vetusto alfabeto que Domingo Bravo propusiera hace 70 años para la variedad regional quechua de Santiago del Estero, el cual no solo fue diseñado defectuosamente, sino que responde a un criterio claramente castellanizante (Andreani, 2018, p. 6). Para estas personas, sin ninguna formación lingüística, la mayoría monolingües en castellano, el problema es con cuál alfabeto escribir, no la discriminación lingüística, la ausencia de la lengua en el sistema educativo, el peligro de la extinción, la vulneración de los derechos lingüísticos, el drama socio-económico de los hablantes, la violencia de las fuerzas de seguridad al servicio de los terratenientes ni la falta de trabajo. El fanatismo que los anima, los lleva a rechazar los trabajos de documentación que con tanto esfuerzo se vienen realizando e incluso utilizan sus influencias para bloquear algunas iniciativas y propuestas de revitalización.

Hay otro aspecto preocupante, y es que algunos hablantes visualizan como "dirigentes" a quienes somos responsables de las 
cátedras universitarias y se autocalifican o identifican como "las bases", estableciendo un claro paralelismo con el accionar de las organizaciones políticas. Investigadores y docentes tenemos claro que las decisiones respecto del destino de su propia lengua son atribuciones exclusivas de sus hablantes, quienes, en última instancia, decidirán preservarla o permitir su extinción. La universidad solo acompaña y procura promover actitudes lingüísticas positivas en la sociedad hacia la lengua indígena y (re)crear espacios de relevancia en donde ésta pueda desarrollarse. Urge realizar una revisión autocrítica de nuestras prácticas, tanto colectivas como individuales, para desactivar estas miradas que nos transforman en involuntarios referentes de procesos cuya conducción debe estar en manos de la comunidad.

\section{La escuela como centro de promoción}

Indudablemente, uno de los aspectos más importantes en la revitalización lingüística lo constituye la enseñanza de la lengua indígena en las escuelas. Es verdad que esta continúa siendo una asignatura pendiente por parte del Estado, pero no es menos cierto que la creciente actividad que se observa en las escuelas, impulsada por los propios maestros, más temprano que tarde terminará desencadenando las decisiones políticas necesarias. La concientización del docente sobre la necesidad de preservar la lengua es un elemento crucial para que las escuelas se conviertan en centros promotores de la misma. Un notable ejemplo lo constituye la Escuela No734 de Los Cerrillos, en el dpto. Salavina, en donde todo el personal escolar, directivo, docente y auxiliar, estimulan en los niños el uso de la lengua vernácula, mediante actividades lúdicas, representaciones teatrales y elaboración de textos, entre otras actividades, sin imposición alguna, y han logrado erradicar del aula la vergüenza y el estigma de hablar una lengua discriminada. Sin ninguna intervención gubernamental, 
esta escuela promueve el mantenimiento de la lengua al lograr que los padres hablen con sus hijos en quichua asegurando su transmisión intergeneracional. Y lo interesante de este caso, es que no ha habido ninguna intervención externa directa, las acciones se disparan por contagio motorizadas por la lealtad lingüística de los docentes quichuahablantes. No negamos la importancia del rol del gobierno en los procesos de revitalización lingüística, pero no es suficiente con lograr cargos docentes y proyectos de alfabetización si antes no se ha logrado formar docentes capaces de asumir un proceso de educación intercultural bilingüe, que estén preparados para aceptar el pluralismo lingüístico-cultural con el consecuente abandono de toda actitud de homogeneización cultural que cercene el proceso de aprendizaje de la lengua materna, en fin, docentes que tengan la capacidad y la disposición para transicionar desde una concepción pedagógica autoritaria hacia una concepción democrática.

Nuestros egresados realizan como requisito obligatorio para acceder al título, un trabajo de graduación que consiste en una intervención en alguna institución que requiera de la perspectiva intercultural y bilingüe. Aquellas que se han realizado en instituciones educativas, a la par de despertar el entusiasmo en docentes, niños y padres, han generado los insumos y la capacitación docente necesarios para su replicado. Se podrá argumentar que estas experiencias son meros paliativos frente a lo que realmente se necesita: la alfabetización en lengua materna. Pero mientras el desinterés, la desidia y la burocracia de los organismos educacionales sigan siendo una constante, no podemos quedarnos de brazos cruzados. Cuando los niveles directivos de cada escuela involucrada, advierten la importancia de la temática y de lo que se está realizando, transmiten su interés al nivel siguiente, el de supervisión, que es la antesala a los niveles de decisión política. El avance es muy lento, es verdad, pero al menos se realiza sobre la base de convicciones firmes. 


\section{Conclusiones}

La interrupción de la transmisión intergeneracional de la lengua, con lo cual da inicio el proceso de extinción de la misma, es la resultante de un conjunto de factores que pueden ocurrir de manera individual o concurrente. La diversidad de dichos factores es muy amplia y en su momento Fishman (1991, pp. 57-62) propuso su clasificación en categorías a las que denominó "dislocaciones". Frente a esta diversidad, desde la universidad hemos elegido aquellos factores sobre los cuales podemos tener algún grado de incidencia para desarrollar algunas estrategias de revitalización y mantenimiento de la lengua. La morosidad gubernamental en la toma de decisiones políticas que destinen recursos para estos fines, no ha sido óbice para postergar acciones urgentes que hemos encarado con mucho esfuerzo, entre las que acabo de destacar las de documentación de la lengua. Los resultados pueden medirse en términos de un creciente interés por parte de algunos sectores de la sociedad hegemónica y una mayor visualización por parte de los medios de comunicación y las redes sociales. El apoyo constante por parte de las autoridades de la Facultad de Humanidades, Ciencias Sociales y de la Salud de la Universidad Nacional de Santiago del Estero, está permitiendo sostener en el tiempo estas acciones y que no queden como hechos esporádicos y de puro voluntarismo. Nuestra Tecnicatura en EIB año tras año va produciendo egresados con una sólida formación profesional y un espíritu altamente crítico frente a las inequidades económicas y socio-culturales. Los técnicos han podido diseñar y ejecutar intervenciones en variados entornos que han tenido muy buena acogida lo cual, en principio, posibilita su réplica.

Entre las acciones futuras está previsto continuar impulsando proyectos de documentación de esta lengua tratando de recoger léxico especializado, particularmente aquel relacionado con la me- 
dicina popular, con las tareas de agricultura y cría de animales en una economía de subsistencia, con el trabajo "golondrina" de los trabajadores migrantes que se desplazan por el territorio nacional hacia las zonas más productivas (Andreani, 2013) y, también con temas tabúes o que se abordan de manera muy reservada (sexualidad, aborto, incesto, violencia familiar, diversidad de género, etc.).

También se encuentra en curso de tramitación ante el Ministerio de Educación de la Nación, un proyecto de mi autoría para la creación de la carrera Licenciatura en Educación Intercultural Bilingüe, con la Tecnicatura como título intermedio.

Estas son, a grandes rasgos, las acciones de revitalización que se llevan a cabo en la provincia de Santiago del Estero desde la institución universitaria a la que pertenezco.

\section{Referencias bibliográficas}

Albarracín, L.I. (2009). La quichua. Vol. 1. Gramática, ejercicios y diccionario castellano-quichua. Buenos Aires: Dunken.

(2011). La quichua. Vol. 2. Gramática, ejercicios y diccionario quichua-castellano. Buenos Aires: Dunken.

(2013). Los archivos en la historia lingüística del quichua de Santiago del Estero. En VI Jornadas Internacionales de Filología y Lingüistica y I Jornadas de Crítica Genética "Las lenguas del archivo". La Plata, provincia de Buenos Aires: Facultad de Humanidades y Ciencias de la Educación, Universidad Nacional de La Plata.

(2016). La quichua. Vol. 3. Gramática, ejercicios y selección de cuentos. Buenos Aires: Dunken.

(2017). Nuevo Diccionario Temático Quichua-castellano Castellano quichua. Tucumán: Humanitas, Universidad Nacional de Tucumán.

(2018). La Tecnicatura Superior en EIB: una lenta erosión en la sociedad hegemónica. En II Simposio Internacional de Lenguas y Culturas Indígenas de América Latina (ILCLA). Columbus, Ohio, USA: The Ohio State University. 
Albarracín, L.I., Tebes, M.C., \& Alderetes J.R. (Comps) (2002). Introducción al quichua santiagueño por Ricardo L. J. Nardi. Buenos Aires: Dunken.

Alderetes, J. (2001). El quichua de Santiago del Estero. Gramática y vocabulario. Tucumán: Facultad de Filosofía y Letras, Universidad Nacional de Tucumán. (2011). Los procesos de reetnización y su impacto sobre las políticas lingüísticas. En Coronel-Molina, S.M., y McDowell, John H. (Eds.), Proceedings of the First Symposium on Teaching Indigenous Languages of Latin America (pp.82-93). Bloomington, Indiana, USA: CLACS \& MLCP, Indiana University Bloomington \& Association for Teaching and Learning Indigenous Languages of Latin America (ATLILLA).

Alderetes, J.R., \& Albarracín L.I. (2004). El quechua en Argentina: el caso de Santiago del Estero. International Journal of the Sociology of Language, 167, 83-93. Berlín: Walter de Gruyter.

Andreani, H.A. (2013). Migración, maíz y silencio. Aproximaciones al bilingüismo (quichua-español) de los trabajadores 'golondrina' de Santiago del Estero (Argentina). Gazeta de Antropología, 29(1), artículo 02.

(2018). Para qué un alfabeto quichua (en quichua). Revista Trazos Universitarios, diciembre. Santiago del Estero: Universidad Católica de Santiago del Estero. Recuperado de: http://revistatrazos.ucse.edu.ar/index. php/2018/12/06/alfabeto-quichua-quichua/.

Bravo, D. (1956). El quichua santiagueño, reducto idiomático argentino. Tucumán: Universidad Nacional de Tucumán.

(1977). Diccionario Castellano-Quichua Santiagueño. Buenos Aires: Eudeba.

Fishman, J. (1991). Reversing Language Shift. Clevedon, Uk: Multilingual Matters.

Nardi, R. (2002). Introducción al Quichua Santiagueño. En Albarracín, L., Tebes, M. y Alderetes, J. (Comps.). Buenos Aires: Dunken.

Sueldo, B.A. (2014). Experiencia de Revitalización Lingüística en Frías, Santiago del Estero. En Malvestitti, M. y Dreidemie, P. (Comps.), III Encuentro de Lenguas Indígenas Americanas (ELIA)- Libro de Actas. (pp. 553-557). Viedma: Universidad Nacional de Río Negro.

Vigil Oliveros, N. (2004). Acciones para desarrollar la escritura de las lenguas indígenas. Glosas didácticas: Revista electrónica internacional de didáctica de las lenguas y sus culturas, 12, 174-183. 